\title{
New bitongling (NBTL) ameliorates rheumatoid arthritis in rats through inhibiting JAK2/STAT3 signaling pathway
}

\author{
Xiang Li, ${ }^{1,2}$ Yu Xie, ${ }^{2}$ An Kang, ${ }^{3}$ Yue Wang ${ }^{1,2}$ \\ ${ }^{I}$ Nanjing University of Chinese Medicine; Affiliated Hospital of Nanjing University of Chinese Medicine, Jiangsu \\ Province Hospital of Chinese Medicine, Nanjing \\ ${ }^{2}$ Jiangsu Province Hospital of Chinese Medicine, Nanjing \\ ${ }^{3}$ School of Pharmacology, Nanjing University of Chinese Medicine, Nanjing, China
}

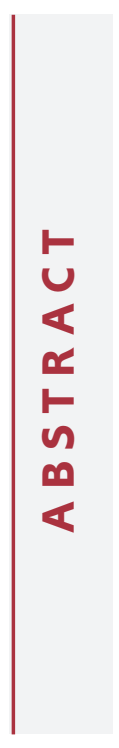

\begin{abstract}
Rheumatoid arthritis (RA) is featured by a variety of physical symptoms and fibroblast-like synoviocytes (FLSs) abnormal proliferation. Increasing evidence has demonstrated that traditional Chinese medicine exerts an important role in RA treatment. New bitongling (NBTL) as one of the traditional Chinese medicine has been reported to be involved in the progression of RA, but the exact mechanism is unclear. In our study, we intended to investigate the effect of NBTL on RA to identify the mechanisms related to JAK2/STAT3 signaling pathway. Extracts of Tripterygium wilfordii (TW), a traditional Chinese herbal medicine, have been widely used for treating RA in China for several decades, so, TW was used as a positive control drug for TBNL. RA rats were constructed by immunization with collagen type II to evaluate the action of NBTL in vivo. Body weight and arthritic index were evaluated. Hematoxylin \& Eosin staining was performed to analysis the morphological changes of ankle joints tissue. TUNEL and flow cytometry were performed to examine cell apoptosis, while CCK8 and Ethynyl-2'-deoxyuridine (EdU) were performed to examine cell proliferation. In addition, the markers of inflammation were detected by Western blot, ELISA, and RT-qPCR. Firstly, we find that rats treated with NBTL or TW not only reduced swelling degree and bone destruction, but also repressed IL- $1 \beta$ and IL- 6 levels . In addition, NBTL and TW could increase the weight of rats, and promote the level of IL-10 and IL-4 in vivo. Furthermore, NBTL inhibited inflammation of FLS, induced cell apoptosis and hindered cell proliferation, which was reversed by dipeptidyl peptidase (DPP), a JAK2/STAT3 pathway activator. Taken together, NBTL potentially retarded RA via JAK2/STAT3 pathway, highlighting novel mechanisms associated with RA
\end{abstract}

Key words: Rheumatoid arthritis; NBTL; JAK2/STAT3; FLS; inflammation.

Correspondence: Dr Yue Wang, Nanjing University of Chinese Medicine; Affiliated Hospital of Nanjing University of Chinese Medicine, Jiangsu Province Hospital of Chinese Medicine, Nanjing, No. 155, Hanzhong Road, Qinhuai District, Nanjing, China.

E-mail: wangyuephd@126.com

Contributions: YW, conceived and designed the study; XL,YX, AK, performed the literature search and data extraction; XL, drafted the manuscript. All the authors have read and approved the final version of the manuscript and agreed to be accountable for all aspects of the work.

Conflict of interest: The authors declare that they have no competing interests, and all authors confirm accuracy.

Availability of data and materials: All data generated or analyzed during this study are included in this published article.

Ethical Approval: The experimental protocol was established, according to the ethical guidelines of the Helsinki Declaration and was approved by the Animal Ethics Committee of Nanjing University of Chinese Medicine. 


\section{Introduction}

Rheumatoid arthritis (RA), a chronic disease related to inflammation and autoimmunity, mainly disturbs joints. If treated inappropriately, it may lead to joint injury and even irreversible disability. ${ }^{1}$ The global incidence of RA is rising, affecting $0.5 \%$ to $1 \%$ of the total population, with a ratio of $3: 1$ (female-to-male). ${ }^{2}$ There may be significant differences between affected individuals in the progress and etiology of RA, and its treatment is still challenging. Although RA treatment has improved significantly, as many as $30 \%$ of the patients did not respond to the interventions or had significant side effects related to treatment. ${ }^{3}$

Fibroblast-like synoviocytes (FLSs) are the most common cell type on pannus-cartilage junction in RA. FLS may eventually cause joint destruction by generating cytokines, chemical factors and matrix degrading molecules. ${ }^{4}$ In addition, FLSs with aggressive phenotype can resist apoptosis, proliferate excessively, and invade surrounding tissues like bones and cartilage, thus leading to angiogenesis, activating immune cells and arthritis. ${ }^{5}$ Therefore, understanding the mechanism of FLS in RA is crucial to the treatment of RA. ${ }^{6}$

Traditional Chinese medicine assumes that RA is caused by blood stagnation, heat and toxins. ${ }^{7}$ New bitongling (NBTL) prescription was derived from the experience and thoughts of L.Q. Wang (1919-1999), the late professor of Nanjing University of Traditional Chinese Medicine, a well-known Chinese medicine expert in rheumatology (composed and effective compositions of NBTL were showed in Table 1). In the preliminary study, the research group used orthogonal T-value method to analyze and study the interaction and dose of the main drugs and auxiliary drugs of the compound, simplified the compound, and finally determined to be composed of Herba Ephedrae, Ramulus Cinnamomi, Caulis Sinomenii, Radix Saposhnikoviae, Radix Aconiti Preparata, Nidus Vespae. ${ }^{8}$ Early research study found that the NBTL can be effective treatment of RA, ${ }^{9}$ significantly inhibited the overexpression of vascular endothelial growth factor (VEGF) mRNA in synovial tissue of joints, ${ }^{10}$ and inhibited the increase of soluble intercellular adhesion factor -1(sICAM-1) in serum of C57BL mice. ${ }^{11}$ In addition, the microvascular density and the overexpression of IL-17 in the synovial tissue of the joint of the model rats were decreased by NBTL. ${ }^{12}$ It is reported that NBTL inhibits the expression of prostaglandin E2 (PGE2), have antiinflammatory effects, ${ }^{13}$ can be different levels of the two aspects of nonspecific immunity and nonspecific immune inhibition, ${ }^{14}$ can effectively reduce rats serum and synovial tissues of IL-2, the content of TNF alpha, ${ }^{15}$ but the exact mechanism is unclear. Therefore, this study sought to determine whether NBTL has therapeutic effect on RA and study the therapeutic mechanism. In addition, Clinical practice guideline for the use of the extracts of Tripterygium wilfordii (TW) in the treatment of RA was approved on June 2020 by the Standardization Office of Chinese Association of Chinese Medicine. ${ }^{16,17}$ So, TW was used as a positive control drug for TBNL. This study uses a collagen-induced arthritis (CIA) rat model to assess the therapeutic effect of NBTL on RA. This study also observed the effects of NBTL on proliferation and apoptosis of FLS. This study investigated whether NBTL treatment could restore the immune balance and apoptosis of FLS in RA.

\section{Materials and Methods}

\section{Rheumatoid arthritis induction}

Adult female Sprague-Dawley (SD) rats (200-250 g) were used, 7-10 weeks. SD rats were acquired from Zhejiang Academy of Medical Sciences (Zhejiang, China). Rats were kept in standard animal rooms with temperatures of $18-22^{\circ} \mathrm{C}$, where food and water are freely available. Rats were treated humanely using approved procedures granted by the local Animal Ethics Committee. Collagen-induced arthritis (CIA) was caused in SD rats by immunization with collagen type II (CII, 234184-M, Sigma-Aldrich, St. Louis, MO, USA). ${ }^{18}$ In short, collagen was dissolved in $0.1 \mathrm{M}$ acetic acid $(2 \mathrm{mg} / \mathrm{mL})$ and gently stirred overnight at $4{ }^{\circ} \mathrm{C}$. Emulsification contained the same volume of Complete Freund's Adjuvant (CFA, F5881, Sigma-Aldrich) containing mycobacteria to produce inducers. Each rat was injected subcutaneously with $100 \mu \mathrm{L}$ emulsion at the bottom of the tail. The second injection was performed 14 days after immunization to stabilize the disease symptoms.

\section{Grouping of animals}

There were 6 groups (6 rats per group): Control group; RA group (Collagen induced arthritis); TW group (RA+treated with TW $8 \mathrm{mg} / \mathrm{kg}$ body weight oral daily); RA+NBTL (low concentration) group (RA+treated with NBTL $2.75 \mathrm{~g} / \mathrm{kg}$ body weight oral daily); RA+NBTL (medium concentration) group (RA+treated with NBTL $5.5 \mathrm{~g} / \mathrm{kg}$ body weight oral daily); RA+NBTL (high concentration) group (RA+treated with NBTL $11 \mathrm{~g} / \mathrm{kg}$ body weight oral daily). The dosage was selected according to the preliminary research conducted in laboratory and literature. The arthritis index of these animals was evaluated using a scale of 0 to 4 points, as follows: 0 , normal; 1 . slight erythema and swelling; 2 . moderate erythema and swelling; 3 . severe erythema and swelling, limited joint use; 4 , deformed claws. ${ }^{19}$ The two hind legs both were scored in each rat, with the highest possible score being 8 points. ${ }^{19}$

\section{ELISA}

The level of Interleukin-6 (IL-6), Interleukin-1 $\beta$ (IL-1 $\beta$ ), Interleukin-10 (IL-10), and Interleukin-4, (IL-4) were measured according to the instructions of a commercial ELISA kit (Nanjing Jiancheng, Bioengeering Institute, Nanjing, China).

\section{H\&E staining}

After anesthesia and sacrifice on the $33^{\text {rd }}$ day after immunization, ankle joints of rats were collected, fixed in $4 \%$ paraformaldehyde for $24 \mathrm{~h}$, dehydrated with graded alcohol and cleared in xylene, paraffin embedded, and sectioned $(5 \mu \mathrm{m})$. Sections were dewaxed and rehydrated. Then, sections were stained with hematoxylin (474-07-7, Jisskang Biotechnology Co., Ltd., Qingdao, China), and then eosin (RY0648, Jisskang), followed by dehydration and mounting. The morphological changes of FLS in the rat ankle joints sections were subjected to a morphological image analysis system (JD801; Jeda Technology Co. Ltd., Nanjing, China).

Table 1. Components and the effective compositions of NBTL.

\begin{tabular}{lc} 
Component & Weight (g) \\
Herba Ephedrae & 6 \\
Caulis Sinomenii & 15 \\
\hline Ramulus Cinnamomi & 10 \\
Nidus Vespae & 10 \\
Radix Saposhnikoviae & 10 \\
Radix Aconiti & 10 \\
\hline
\end{tabular}




\section{qRT-PCR}

TRIzol reagent (Takara, Liaoning, China) was used to extract the total RNA. M-MLV Reverse Transcriptase (RNase H) kit (Takara, Liaoning, China) was performed to synthesize cDNA. qPCR was performed with SYBR Green qPCR kit (Life Technologies, Shanghai, China). $\beta$-actin ${ }^{20}$ serves as an endogenous control for MCM7, ${ }^{21} \mathrm{PCNA},{ }^{22} \mathrm{Ki}-67,{ }^{23} \mathrm{Bcl}-2$, Bax, ${ }^{24}$ caspase- 3 and caspase- $9 .{ }^{25}$ The primers are listed in Table 2.

\section{Western blot}

Tissues or cell lysates were isolated using cell lysis buffer (Beyotime, Nanjing, China). The membrane was transferred and blocked at $37^{\circ} \mathrm{C}$ for $1 \mathrm{~h}$, followed by primary antibodies incubation, rabbit anti-MCM7 (1:1000, ab52489), PCNA (1:2000, ab92552) and Ki-67 (1:1000, ab16667), Bax (1:5000; ab32503), Bcl-2 (1:1000, ab59348), Cleaved caspase-3 (1:1000, ab49822), Cleaved caspase-9 (1:1000, ab2324), JAK2 (1:5000, ab108596), pJAK2 (1:5000, ab32101), STAT3 (1:2000, ab109085), p-STAT3 $(1: 2000, \mathrm{ab} 76315)$ and rabbit anti- $\beta$-actin $(1: 2000$, ab8227) were purchased from Abcam (Cambridge, UK), and then re-probed with immunoglobulin $\mathrm{G}$ (IgG) complexed to horseradish peroxidase (1:2000, ab6721) antibody. The chemiluminescence kit (Millipore, Germany) was adopted to observe the immune response zone and Image J software (Image J Software Inc., USA) was used to quantify the integrated density of each band. Antibodies mentioned before were supplied by Abcam (Cambridge, UK).

\section{Primary FLS isolation}

Discarded isolated synovial tissue were obtained. Tissue was cut into small pieces. Samples were then digested with $0.4 \%$ type I collagenase for $2 \mathrm{~h}, 0.25 \%$ trypsin for $30 \mathrm{~min}$. Digestion were performed in a $37^{\circ} \mathrm{C}$. Filtered the digested cells using a $70 \mathrm{~mm}$ strainer and washed three times with PBS. Using immunocytochemistry staining with Anti-Vimentin antibody and flow cytometry with Anti-CD55 antibody for cell purity test. ${ }^{19,24}$

\section{CCK8 assay}

CCK8 assay kit (CK04, Dojindo, Japan) was utilized to analyze cell viability. Cells were incubateds with different dosages of $\operatorname{NBTL}(10,20,30 \mu \mathrm{M})^{26}$ and TW $(50 \mathrm{nM})$ concentrations for $48 \mathrm{~h}$. $1 \times 10^{4}$ cells/well were seeded for $24 \mathrm{~h}$ of pre-culture, followed by $48 \mathrm{~h}$ treatment. After NBTL or TW treatment, $10 \mu \mathrm{L}$ CCK8 was added for reaction at $37^{\circ} \mathrm{C}$ for $3 \mathrm{~h}$. After that, optical density at 450 $\mathrm{nm}$ was checked on a microplate reader. A cell growth curve was drawn.

\section{TUNEL assay}

Tissue samples were fixed at $37^{\circ}$ for $30 \mathrm{~min}$, then incubated with $0.2 \%$ Triton X-100 for $5 \mathrm{~min}$. In order to improve the permeability, samples were incubated with $50 \mu \mathrm{L}$ reaction mixture (the ratio of concentrated enzyme solution to label solution is 1:9) at $37^{\circ} \mathrm{C}$ for $1 \mathrm{~h}$ and observed using a fluorescence microscope. Randomly selected five fields to determine the apoptosis rate, apoptosis rate $=($ apoptotic cells $/$ total cells $) \times 100$.

For cell apoptosis in vitro, $48 \mathrm{~h}$ after NBTL or TW treatment, FLSs were fixed using $2 \%$ formalin at $37^{\circ} \mathrm{C}$ for $1 \mathrm{~h}$, using $0.1 \%$ Triton X-100 to permeate on ice for $2 \mathrm{~min}$, followed by 1 -h-incubation with $50 \mu \mathrm{L}$ TUNEL reaction mixture at $37^{\circ} \mathrm{C}$ under dark conditions. The results were observed under fluorescence microscope (LSM880, Zeiss, Germany). ${ }^{24}$.

\section{Ethynyl-2'-deoxyUridine assay}

Cell proliferation after NBTL or TW treatment was detected using an EdU kit (CA1170, Solarbio, China). In brief, cells were incubated with $50 \mu \mathrm{M}$ EdU for $18 \mathrm{~h}$ firstly. Cells were fixed with $4 \%$ formaldehyde for $30 \mathrm{~min}$, followed by $0.5 \%$ Triton X-100 for ten minutes. Then, incubated $30 \mathrm{~min}$ with $1 \times$ Apollo reagent. Finally, DAPI was used for nuclear staining. ${ }^{27}$

\section{Flow cytometry}

$1.00 \times 10^{6}$ cells $/ \mathrm{mL}$ were applied for propidium iodide staining for 10 min and subjected to cell apoptosis analysis using FITC and FACSCalibur flow cytometer (342975, BD, New Jersey, USA). The number of cells was acquired at $488 \mathrm{~nm}$ wavelength and analyzed using Cell Quest software.

\section{Statistical analysis}

The mean $\pm \mathrm{SD}$ represents data from three independent experiments. SPSS 21.0 (IBM Corp., NY, USA) is used for statistical analysis of all data. Data within multiple groups is analyzed using one-way ANOVA and Tukey's post-tests. Time-based multi-comparison was tested by repeated measures ANOVA; $\mathrm{p}<0.05$ indicated that the difference is statistically significant.

\section{Results}

\section{NBTL impacts inflammation in RA rats}

In the third week after the induction of RA rats, we analyzed the degree of swelling of the hind paws of rats. Clinical symptoms of RA can be seen in RA rats, including swelling and redness of the hind paws. Compared to rats in the RA group, rats treated with NBTL or TW reduced swelling degree and exhibited NBTL concentration dependence (Figure 1A). NBTL and TW also significantly increased the weight of rats (Figure 1B) while reducing the arthritis index value (Figure 1C), which also showed NBTL concentration dependence.

The serum levels of IL-1 $\beta$, IL-6, IL-10, IL-4 were analyzed by ELISA, which indicated a significant increase in the expression of IL-1 $\beta$ and IL- 6 in rats of RA group, relative to the control group, while NBTL treatment was able to suppress IL-1 $\beta$ and IL-6 levels with concentration dependence compared to the RA group. In contrast, NBTL and TW treatment significantly promoted the level of IL-10 and IL-4 (Figure 1D). Taken together, NBTL could prevent inflammation in RA rats.

Table 2. Primer sequences used for qRT-PCR.

\begin{tabular}{lll} 
Genes & & Primer sequences $\left(5^{\prime}-3^{\prime}\right)$ \\
MCM7 & Forward & GATAAGAGGAAACATCCACATCTG \\
& Reverse & AGACGGTCAATGTAAGACAGG \\
PCNA & Forward & TGACTGGTTATCGTCCCTCCT \\
& Reverse & CTCAAACATGGTGGCGGAGT \\
\hline Ki-67 & Forward & GGGTTTCCAGACACCAGAC \\
& Reverse & CCAGGAAGACCAGTTAGACC \\
Bcl-2 & Forward & GACTGAGTACCTGAACCGGC \\
& Reverse & TAGTCCACAAAGGCATCCCAG \\
\hline Bax & Forward & CCAGTTGAAGTTGCCGTCAGAA \\
& Reverse & GCGAGTGTCTCAAGCGCATC \\
Caspase-3 & Forward & GCTTGTCGGCATACTGTTTCAG \\
& Reverse & AGAACTGGACTGTGGCATTGAG \\
\hline Caspase-9 9 & Forward & ATTGGCGACCCTGAGAAG \\
& Reverse & CCAGATGCTGTCCCATACC \\
$\beta$-actin & Forward & GAGAGGGAAATCGTGCGTGAC \\
& Reverse & CATCTGCTGGAAGGTGGACA \\
\hline
\end{tabular}




\section{NBTL inhibits synoviocyte proliferation in RA rats}

To discover the effect of NBTL in the RA model system, histopathology of each group of rats was assessed. The rats in the control group showed normal joint structure, while the RA model rats exhibited substantial inflammatory cells penetration, synovial hyperplasia, bone destruction. TW and NBTL-mediated dosedependent reduce bone destruction, and synovial hyperplasia of rats (Figure 2A). The mRNA of MCM7, PCNA and Ki-67 was
A

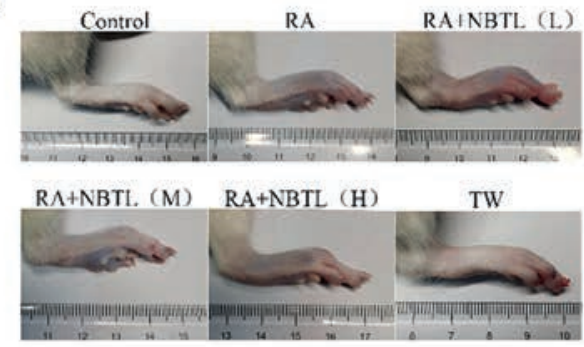

B

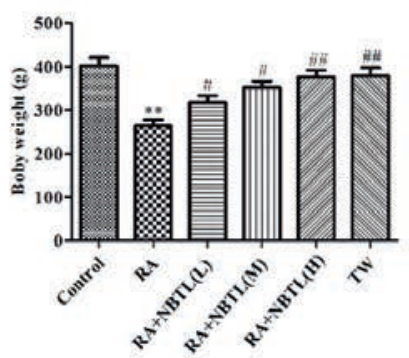

$\mathrm{C}$

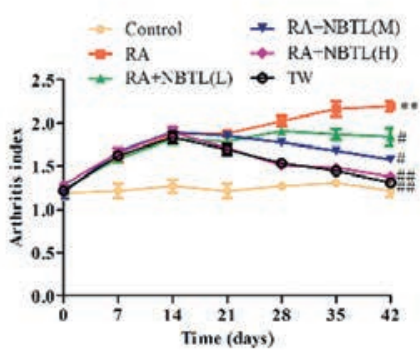

D
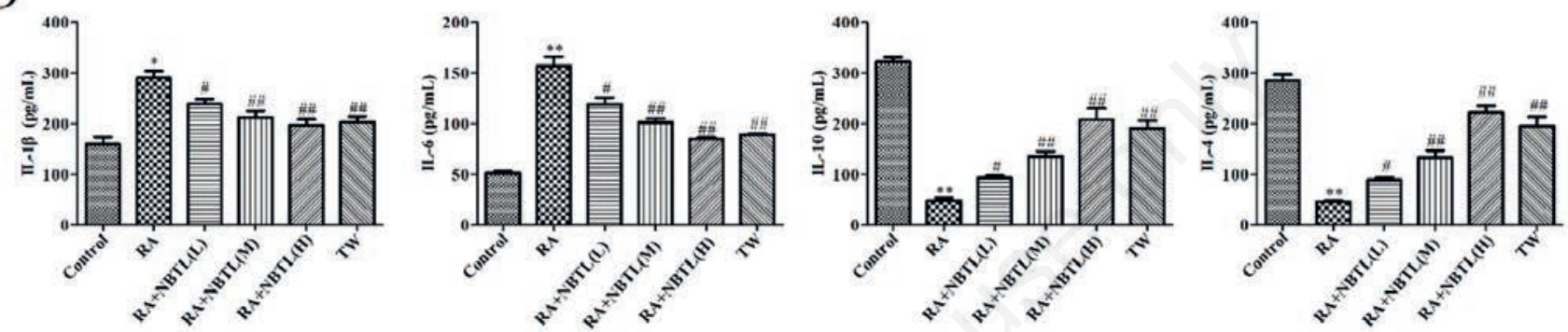

Figure 1. Inflammation inhibitory effects of NBTL on rheumatoid arthritis (RA) in vivo. RA rats were constructed by immunization with collagen type II, then we treated RA rats with different concentrations of NBTL, and TW (positive control drug for NBTL). A) Observation of the paw swelling. B) Body weight of rats in treated groups. C) Arthritic scores of rats were used to assess the severity of the disease. D) ELISA was performed to analyze the serum levels of IL-1 $\beta$, IL-6, IL-10, and IL-4. Data were shown as mean \pm SD (n = 10 for all groups). ${ }^{*} \mathrm{p}<\mathbf{0 . 0 5},{ }^{* *} \mathrm{p}<\mathbf{0 . 0 1}$ vs Control, ${ }^{\#} \mathrm{p}<\mathbf{0 . 0 5},{ }^{\# \#} \mathrm{p}<\mathbf{0 . 0 1}$ vs $\mathrm{RA}$.

A
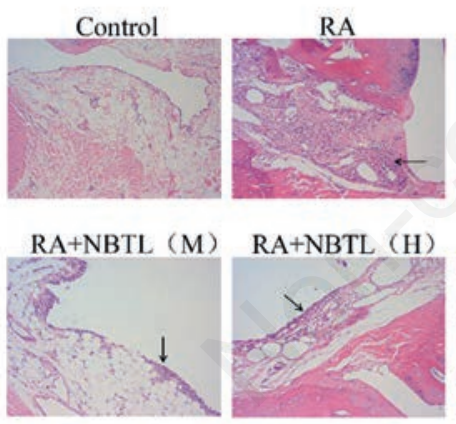

RA+NBTL (L)

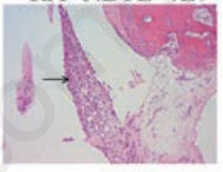

TW

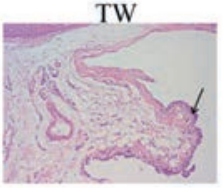

B

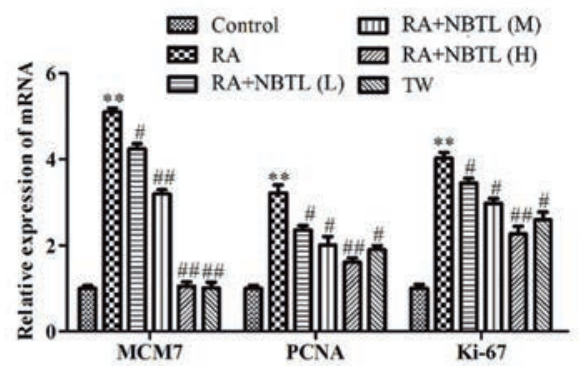

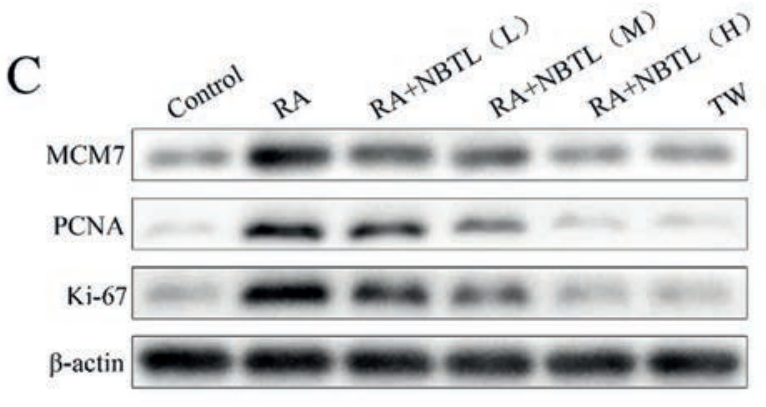

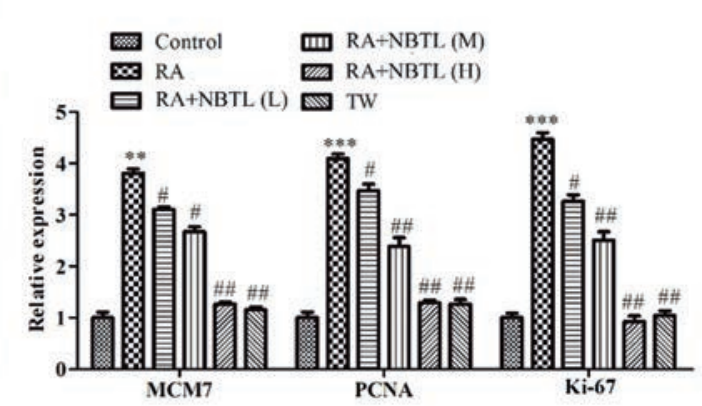

Figure 2. NBTL inhibits cell proliferation in RA in vivo. A) Damage to joint tissues determined using $\mathrm{H} \& \mathrm{E}$ staining $(200 \times)$. B) The mRNA expression levels of MCM7, PCNA and Ki-67 were measured by qRT-PCR. C) The protein expression of proliferation-associated proteins MCM7, PCNA and Ki-67 were measured by Western blot. Data were shown as mean \pm SD ( $n=10$ for all groups). ${ }^{* *} p<0.01$, ${ }^{* * *} \mathrm{p}<0.001$ vs Control, \# $\mathrm{p}<0.05,{ }^{\# \#} \mathrm{p}<0.01$ vs RA. 
assessed using qRT-PCR. The results exhibited that mRNA of MCM7, PCNA and Ki-67 were significantly promoted in RA group, respectively, but were significantly repressed by TW and NBTL (Figure 2B). Finally, the expression of proliferation-associated proteins MCM7, PCNA and Ki-67 were detected using Western blot. The results exhibited that MCM7, PCNA, Ki-67 in RA group were significantly promoted. Conversely, MCM7, PCNA and Ki-67 were remarkably diminished due to TW and NBTL treatment (Figure 2C). Collectively, NBTL inhibited cell proliferation in RA rats.

\section{NBTL inhibits inflammation of fibroblast-like synovio- cytes (FLS) in vitro}

For in vitro experiments, FLS was initially characterized by immunofluorescent staining. These cells were Vimentin-positive (Figure 3A). The expression of CD55 in cells was also determined using flow cytometry assay (Figure 3B), which clarified that FLS purity $>90 \%$, making it suitable for subsequent experiments. Then, after treating FLS with NBTL, the level of inflammation-related factors after NBTL treatment were measured by ELISA. The result

A
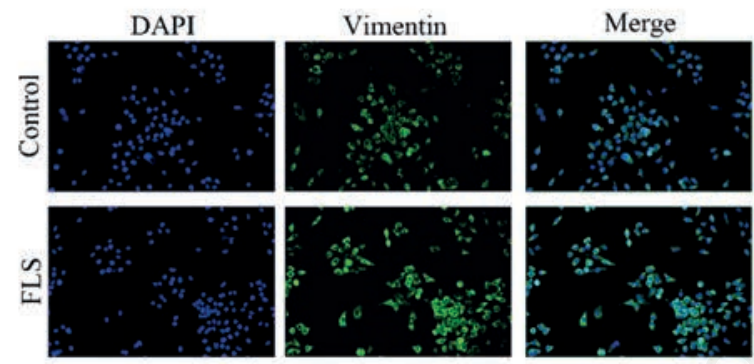

B
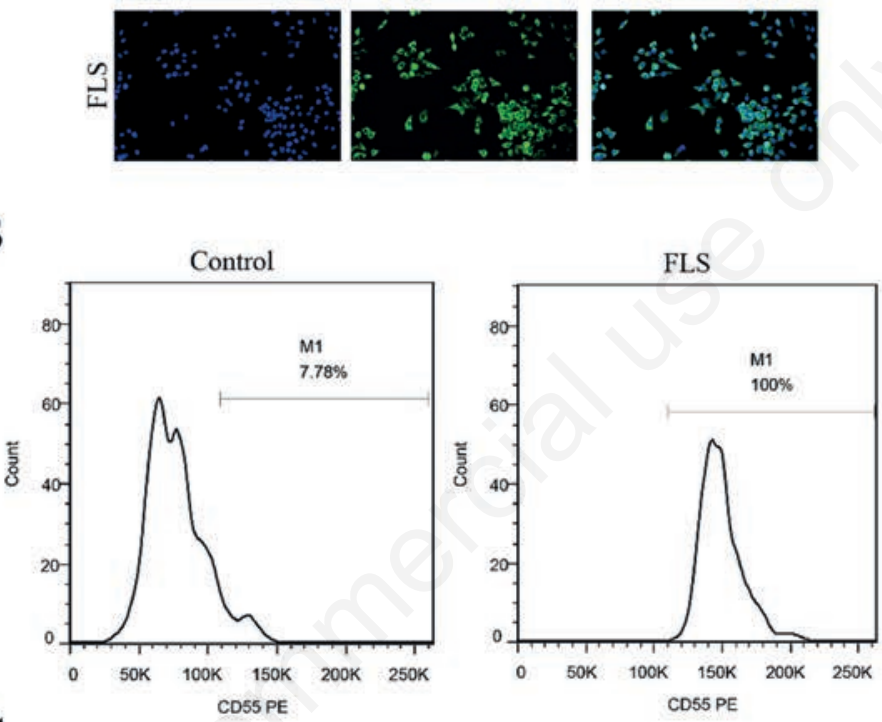

C
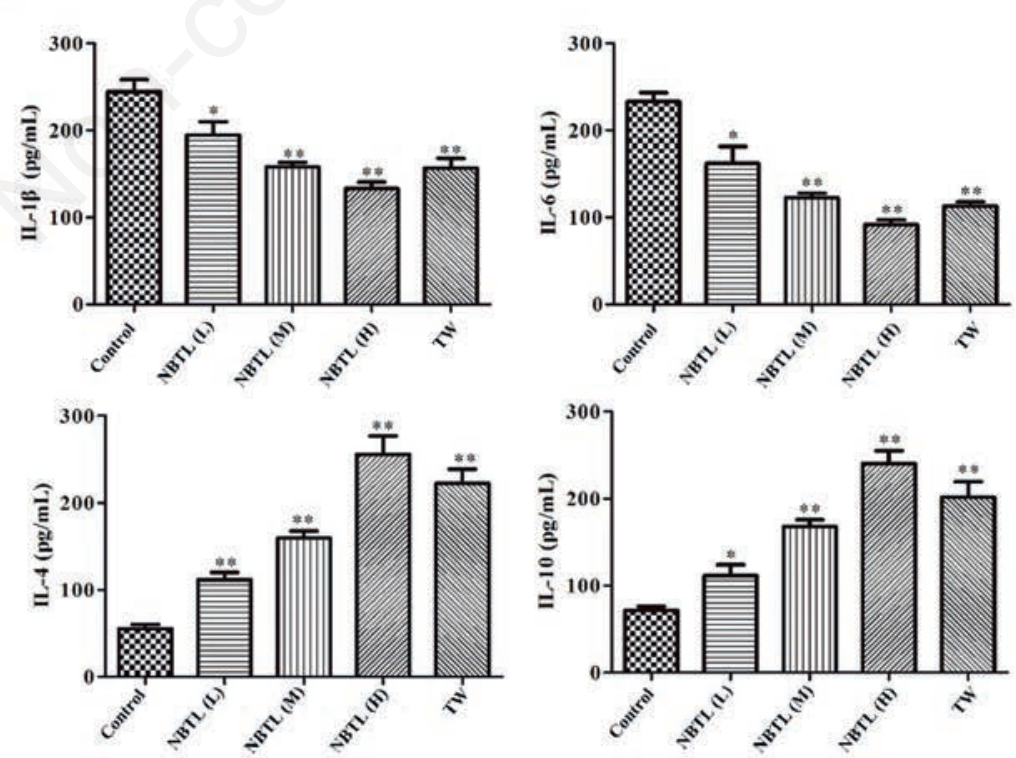

Figure 3. The validation and inflammation of fibroblast-like synovial (FLS) cells isolated from RA rats treated with NBTL. A) The expression levels ofv were determined using immunocytochemistry $(200 \times)$. B) The positive cell rate of CD55 were determined using flow cytometry. C) ELISA was performed to analyze the supernatant levels of IL-1 $\beta$, IL-6, IL-10, and IL-4 from FLS cells. Data were shown as mean \pm SD $(\mathrm{n}=3) .{ }^{*} \mathrm{p}<0.05,{ }^{* *} \mathrm{p}<0.01$ vs Control. 
indicated that IL-1 beta and IL-6 were significantly reduced after NBTL treatment, while the level of IL-10 and IL-4 were significantly promoted, in a concentration-dependent manner (Figure 3C). Collectively, NBTL might inhibit the inflammation of FLS.

NBTL induces FLS cell apoptosis and hinders proliferation in vitro

In the following experiments, we detected FLS apoptosis by TUNEL assay, which indicated that apoptosis rate was significantly promoted after NBTL treatment with concentration dependence (Figure 4A). Moreover, we detected proliferation of FLS cells through CCK8 (Figure 4B) and EdU staining (Figure 4C), and both showed that NBTL significantly restrained cell proliferation. In contrast to the proliferation results, subsequent results of flow cytometry analysis were consistent with the TUNEL assay results (Figure 4D).

Next, the mRNA of Bcl-2, Bax, caspase- 3 and caspase- 9 were detected by qRT-PCR, which demonstrated that the mRNA of Bax, caspase- 3 and caspase- 9 were significantly improved after NBTL treatment, while the results of Bcl-2 were the opposite (Figure 4E). Same as mRNA, the result of Western blot indicated that in the group treated with NBTL, Bax, Cleaved caspase-3, and Cleaved caspase-9 increased significantly, while Bcl-2 was suppressed (Figure 4F). Taken together, NBTL might stimulate FLS apoptosis, while restraining proliferation in vitro.

\section{NBTL's effect on FLS cell proliferation and apoptosis was via JAK2/STAT3 pathway}

Recent studies have validated the significant roles of Janus kinase (JAK)/signal transducer and activator of transcription (STAT) signaling pathways in RA. ${ }^{28}$ So, we assumed that NBTL modulated FLS cells via the JAK/STAT pathway and this hypothesis was validated. First, we used Western blot to measure the level of JAK2/STAT3 axis-related proteins and found that NBTL was able to significantly inhibit p-JAK2 and p-STAT3 level. Then we treated FLS with both NBTL and DPP (JAK2/STAT3 pathway activator) simultaneously and found that p-JAK2 and p-STAT3 level were rescued by DPP (Figure 5A). Above results revealed

A

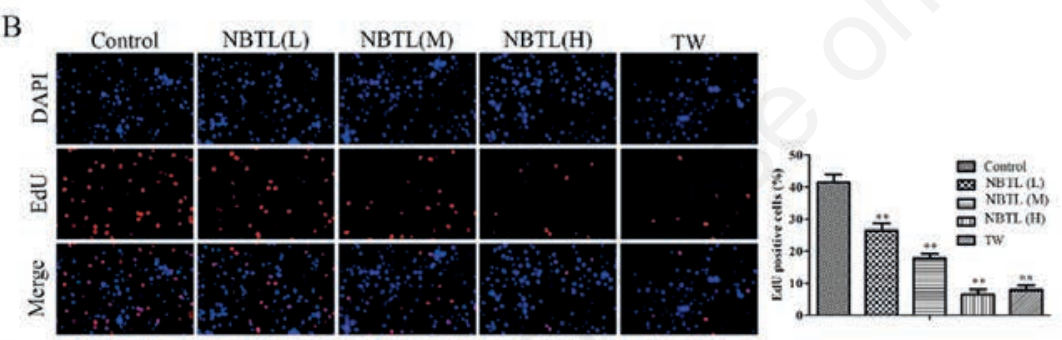

C
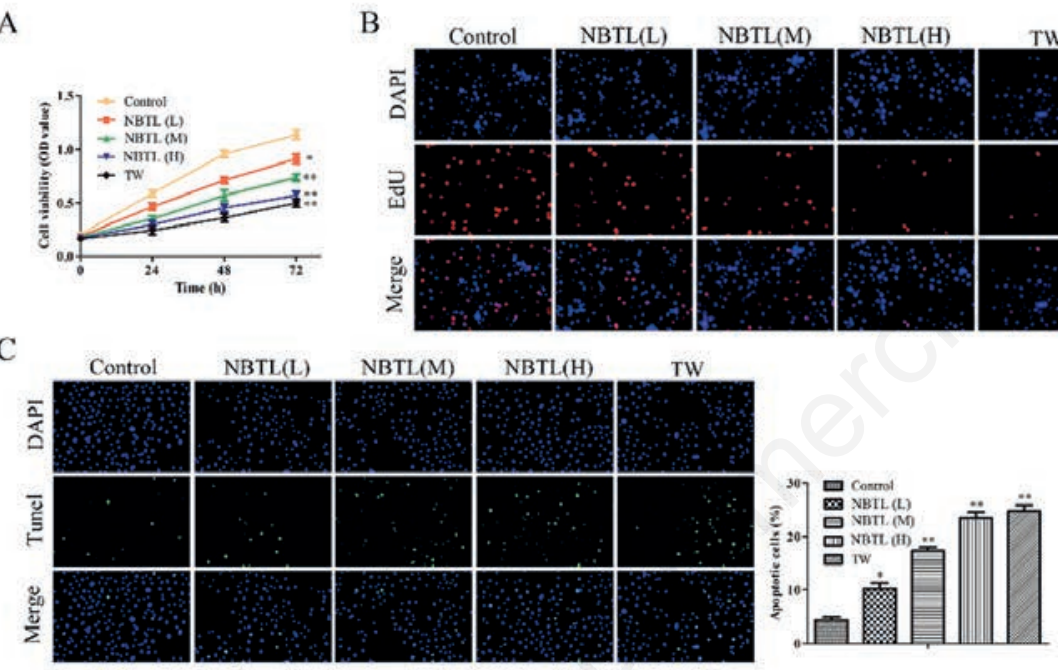

D
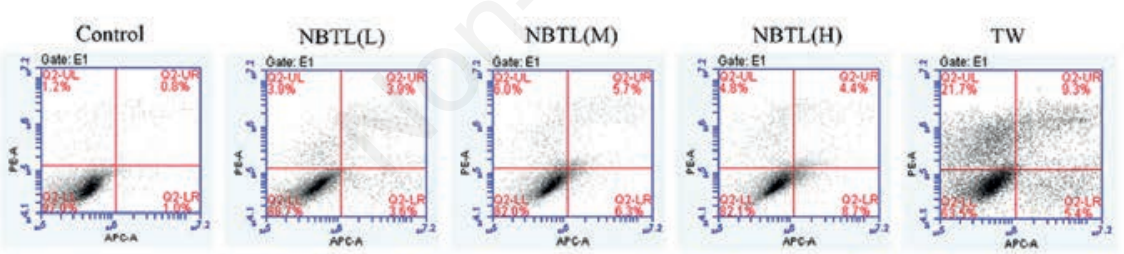

E

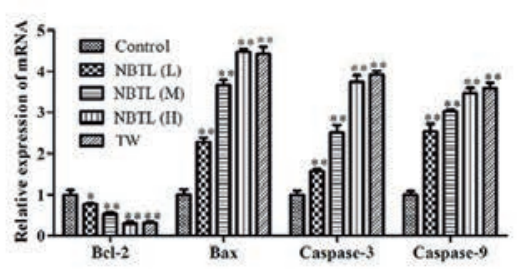

F

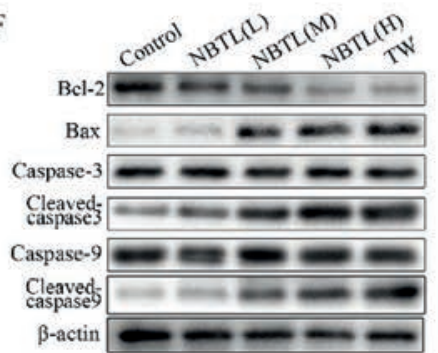

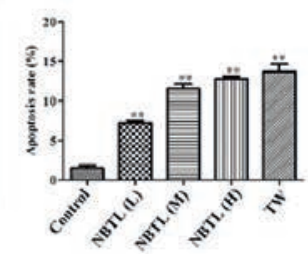

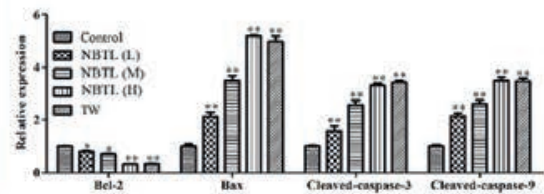

Figure 4. NBTL inhibits cell proliferation and promotes apoptosis of FLS in vitro. A) The cell apoptosis determined using TUNEL staining $(400 \times)$. B) The cell viability was determined using CCK8 assay. C) The cell proliferation was determined using EdU staining $(400 \times)$. D) The cell apoptosis determined using flow cytometry. E) The mRNA expression levels of Bcl-2, Bax, caspase-3 and caspase9 were measured by qRT-PCR. F) The expression of apoptosis-associated proteins, including Bcl-2, Bax, Cleaved caspase-3, Cleaved caspase-9 were measured by Western blot. Data were shown as mean \pm SD ( $n=10$ for all groups). ${ }^{*} \mathbf{p}<0.05,{ }^{* *} \mathrm{p}<0.01$ vs Control. 
that NBTL can suppress the JAK2/STAT3 pathway.

In order to further confirm that NBTL's effect on FLS cell proliferation and apoptosis is through the JAK/STAT pathway, we detected cell proliferation and apoptosis by CCK8 (Figure 5B), EdU staining (Figure 5C), TUNEL staining (Figure 5D), and flow cytometry (Figure 5E), respectively. It was found that NBTL significantly promoted FLS apoptosis and inhibited proliferation, while DPP can reverse NBTL's effects. Finally, the level of Bcl-2, Bax, Cleaved caspase- 3 and Cleaved caspase-9 were measured via Western blot. NBTL resulted in enhanced the expression of Bax, Cleaved caspase- 3 and Cleaved caspase- 9 , and restrained the level of Bcl-2. More importantly, the DPP treatment reversed such activation effects of NBTL on the cell apoptosis (Figure 5F).

\section{Discussion}

RA is a systemic immune disease, which is chronic and progressive and will reduce the patient's life expectancy by 3 to 20 years. The pathogenesis of RA is very complex, so treatment is usually aimed at improving life quality by slowing down disease progression.

CIA is an autoimmune model, which is helpful to study possible therapeutic strategies for injury and inflammation in RA. ${ }^{18,29}$ In this research, the effects of NBTL on RA rats were studied by inducing a CIA model. Animal research showed that compared with RA group, the severity of arthritis decreased significantly after NBTL treatment. Histopathology also indicated that the articular cartilage was intact after NBTL treatment, indicating that arthritis of RA rats was significantly relieved after NBTL treatment. It is reported that inflammation plays a major role in the progression of RA, and IL- $1 \beta$ and IL- 6 plays a proinflammatory role in RA. ${ }^{30,31}$ While IL-10 and IL-4 are anti-inflammatory cytokines. ${ }^{32}$ So, we tested IL-1 $\beta$, IL- 6 , IL-10 and IL-4 to check for the antiinflammatory effects of NBTL. Our study indicated that the contents of IL- $1 \beta$ and IL-6 in RA group were upregulated compared to control group, while repressed after NBTL treatment. In contrast, IL-10 and IL-4 were upregulated after NBTL treatment. Our results showed that NBTL can ameliorate RA by inhibiting the level of inflammatory cytokines. These results confirmed that NBTL had anti-inflammation effect.

At present, it is now well-known that occurrence of RA may be due to the imbalance between proliferation and apoptosis. ${ }^{33}$ FLS is synovial cell which produces synovial fluid. FLS is crucial to the regulation of cartilage and joint damage, deformation and destruction in rheumatoid arthritis. ${ }^{34}$ FLS undergo significant changes in the development of RA, overcoming contact inhibition, and experiencing abnormal proliferation, which lead to tissue damage, even tissue degradation. ${ }^{35}$ Therefore, repressing of FLS proliferation and inducing of cell apoptosis are considered to be a useful strate-
A
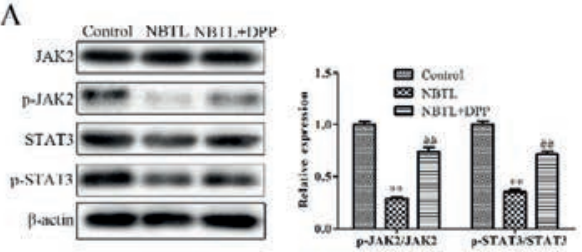

B

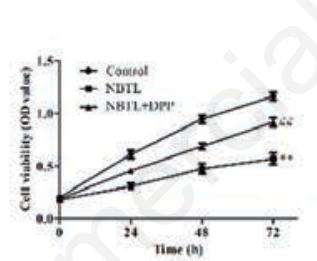

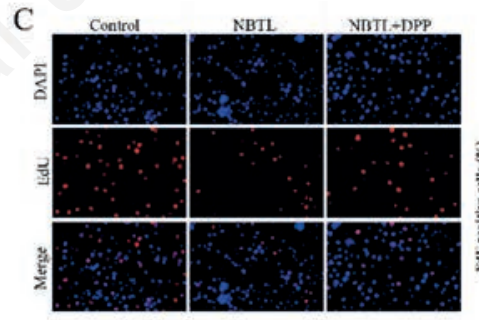

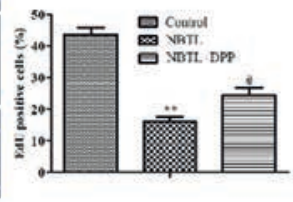

E
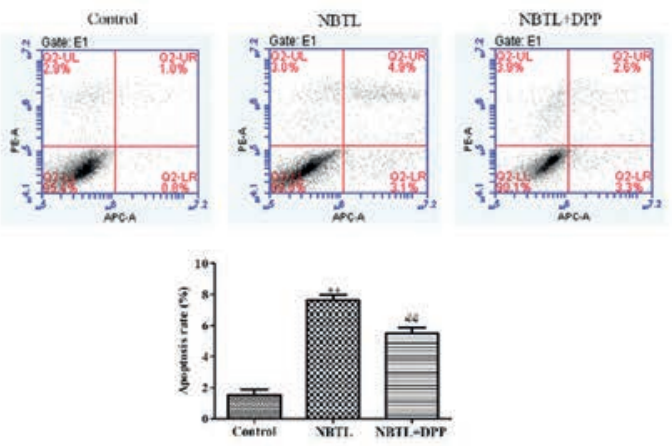

F
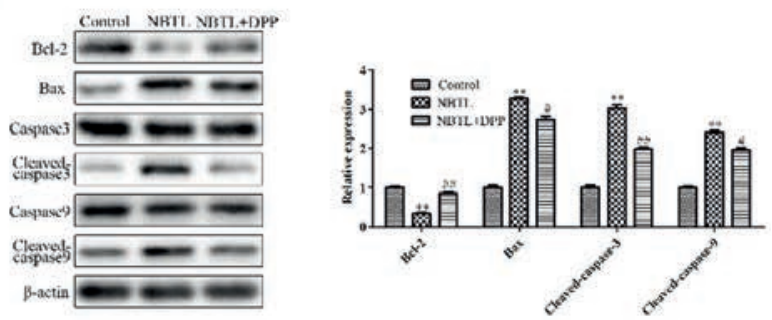

Figure 5. The effect of JAK2/STAT3 pathway on NBTL's impacts on cell proliferation and apoptosis of FLS in vitro. We treated FLS with both NBTL and DPP (JAK2/STAT3 pathway activator) simultaneously. A) The protein expression levels of JAK2/STAT3 pathway were measured by Western blot. B) The cell viability was determined using CCK8 assay. C) The cell proliferation was determined using EdU staining $(400 \times)$. D) The cell apoptosis was determined using the TUNEL assay $(400 \times)$. E) The cell apoptosis was determined using flow cytometry. F) The protein expression levels of apoptosis-associated proteins, including Bcl-2, Bax, Cleaved caspase-3, Cleaved caspase-9, were measured by Western blot. Data were shown as mean $\pm \mathrm{SD}$ ( $\mathrm{n}=10$ for all groups). ${ }^{* *} \mathrm{p}<0.01$ vs $\mathrm{Control},{ }^{\#} \mathrm{p}<0.05$, ${ }^{\#} \mathbf{p}<\mathbf{0 . 0 1}$ vs $\mathbf{C}$. 
gy for the treatment of RA. ${ }^{36,37}$ In present study, our in vitro experiments results showed that NBTL could significantly induced cell apoptosis and hindered proliferation of FLS isolated from RA rat, which is consistent with the results of animal experiments in vivo. It also suggested that NBTL might play its therapeutic effect on rheumatoid arthritis through facilitating apoptosis and restraining proliferation of FLS.

JAK/STAT pathway plays important biological mechanisms, such as cell proliferation and inflammation. JAK/STAT pathway is provoked by receptor binding of cytokines, like IL- $6 .{ }^{38}$ Cells initiate intracellular signal transduction cascade through JAK/STAT pathway stimulated by IL-6, resulting in STAT3 phosphorylation. ${ }^{39}$ Stimulating signals like IL-6 can activate JAK/STAT signaling pathways, and activation of JAK/STAT pathways lead to promoting downstream inflammatory cytokines expression. Elevated level of IL-6 has a positive regulatory effect on the JAK/STAT pathway activation, and this positive feedback results in continuous and expanded inflammatory response in RA. ${ }^{40}$ JAK2 plays a vital role in many immune responses mediated by cytokine, and JAK2 inhibitors have been proved to have significant clinical effects on RA. ${ }^{41}$ STAT3 can be activated by cytokines such as IL6 , and keep activated in RA synovium. ${ }^{42}$ JAK2 and STAT3 are closely associated with RA pathogenesis. Anti-RA drugs usually clarify their mechanism by inhibiting JAK2/STAT3 signaling pathway. ${ }^{28}$ In present study, our results showed that NBTL could significantly inhibit JAK2/STAT3 pathway, and our in vitro experiments further proved that NBTL's effect on FLS cell proliferation and apoptosis was via JAK2/STAT3 pathway.

In summary, in vivo and in vitro experiments carried out in this research all verified that NBTL has therapeutic effects on RA by promoting the apoptosis of FLS and repressing the proliferation of FLS by inhibition of JAK2/STAT3 pathway. In this study, immunology and animal studies are used to support NBTL as a potential drug for RA.

\section{References}

1. Smolen JS, Aletaha D, Barton A, Burmester GR, Emery P, Firestein GS, et al. Rheumatoid arthritis. Nat Rev Dis Primers 2018;4:18001.

2. Safiri S, Kolahi AA, Hoy D, Smith E, Bettampadi D, Mansournia MA, et al. Global, regional and national burden of rheumatoid arthritis 1990-2017: a systematic analysis of the Global Burden of Disease study 2017. Ann Rheum Dis 2019;78:1463-71.

3. Rutherford AI, Patarata E, Subesinghe S, Hyrich KL, Galloway JB. Opportunistic infections in rheumatoid arthritis patients exposed to biologic therapy: results from the British Society for Rheumatology Biologics Register for Rheumatoid Arthritis. Rheumatology (Oxford) 2018;57:997-1001.

4. Bartok B, Firestein GS. Fibroblast-like synoviocytes: key effector cells in rheumatoid arthritis. Immunol Rev 2010;233:233-55.

5. de Oliveira PG, Farinon M, Sanchez-Lopez E, Miyamoto S, Guma M. Fibroblast-like synoviocytes glucose metabolism as a therapeutic target in rheumatoid arthritis. Front Immunol 2019;10:1743.

6. Bi X, Guo XH, Mo BY, Wang ML, Luo XQ, Chen YX, et al. LncRNA PICSAR promotes cell proliferation, migration and invasion of fibroblast-like synoviocytes by sponging miRNA-4701-5p in rheumatoid arthritis. EBioMedicine 2019;50:408-20.

7. Li S, Wang H, Sun Q, Liu B, Chang X. Therapeutic effect of Xuebijing, a traditional Chinese medicine injection, on rheumatoid arthritis. Evid Based Complement Alternat Med
2020;2020:2710782.

8. Jing R, Wang Y. The modernization research on new bitongling compound. Jilin J Tradit Chinese Med 2014;34:225-7.

9. Yuan-Qing G, Yue W. [Clinical study on effect of bitongling granule on treating 30 cases of stagnancy of cold-dampness in the meridian and joints of RA].[Article in Chinese]. Chinese J Inform TCM 2004.

10. Wu S, Wang Y, Xia W. Effect of bitongling granule on VEGF mRNA expression in synovial tissue of collagen-induced rheumatoid arthritis rats. J New Chinese Med 2009;41:98-100.

11. Guo H, Wang Y, Ding W. [Effect of bitongling granule on the expression of serum soluble intercellular adhesion molecule-1 in the C57BL mice induced by inflammation].[Article in Chinese]. Hebei J Tradit Chin Medi 2006;12.

12. Fan R, Wang Y, XU J, Sang F, Song Y, Jiang L. Effect on expression of IL-17mRNA in synovial membrane of collagen induction arthritis by rheumatism painful spirit (bitongling) pellet].[Article in Chinese]. China J Chin Med 2011;5.

13. Feng-Lin Z, Gang W, Fan P, Yan L, Rong-Yue J, Yun-Ke G, et al. [Anti-inflammatory effect of new bitongling compound and its mechanism.[Article in Chinese]. Chin J Experim Tradit Med Formulae 2015.

14. Yan L, Gang W, Fenglin Z, Yunke G, Fang P, Rongyue J, et al. [Xinbitongling on mice immune function].[Article in Chinese]. J Changchun Univ Chinese Med 2015.

15. Shi-Hua Q. [Effects of bitongling particles on IL-2 of serum and tissue in rats with collagen-induced arthritis].[Article in Chinese]. West J Tradit Chinese Med 2011.

16. Zhou YZ, Zhao LD, Chen H, Zhang Y, Wang DF, Huang LF, et al. Comparison of the impact of Tripterygium wilfordii Hook $\mathrm{F}$ and Methotrexate treatment on radiological progression in active rheumatoid arthritis: 2-year follow up of a randomized, non-blinded, controlled study. Arthritis Res Ther 2018;20:70.

17. Chen ZZ, Zhang XD, Chen Y, Wu YB. The role of circulating miR-146a in patients with rheumatoid arthritis treated by Tripterygium wilfordii Hook F. Medicine (Baltimore) 2017;96:e6775.

18. Ahmad Khan M, Sarwar A, Rahat R, Ahmed RS, Umar S. Stigmasterol protects rats from collagen induced arthritis by inhibiting proinflammatory cytokines. Int Immunopharmacol 2020;85:106642.

19. Wang M, Li H, Wang Y, Hao Y, Huang Y, Wang X, et al. Antirheumatic properties of gentiopicroside are associated with suppression of ROS-NF-kappaB-NLRP3 axis in fibroblast-like synoviocytes and NF-kappaB pathway in adjuvant-induced arthritis. Front Pharmacol 2020;11:515.

20. Xiang Y, Yang Y, Lin C, Wu J, Zhang X. miR-23a-3p promoted G1/S cell cycle transition by targeting protocadherin17 in hepatocellular carcinoma. J Physiol Biochem 2020;76:123-34.

21. Chen HY, Tang NH, Lin N, Chen ZX, Wang XZ. Hepatitis B virus $\mathrm{X}$ protein induces apoptosis and cell cycle deregulation through interfering with DNA repair and checkpoint responses. Hepatol Res 2008;38:174-82.

22. Wu S, Huang D, Su X, Yan H, Wu J, Sun Z. Oral exposure to low-dose bisphenol A induces hyperplasia of dorsolateral prostate and upregulates EGFR expression in adult SpragueDawley rats. Toxicol Ind Health 2019;35:647-59.

23. He M, Wang L, Chen Y, Zhang T, Guo J. Effect of Wuziyanzong pill on levels of sex hormones, and expressions of nuclear- associated antigen Ki-67 and androgen receptor in testes of young rats. J Tradit Chin Med 2016;36:743-8.

24. Li GQ, Fang YX, Liu Y, Meng FR, Wu X, Zhang CW, et al. MALAT1-driven inhibition of Wnt signal impedes proliferation and inflammation in fibroblast-like synoviocytes through CTNNB1 promoter methylation in rheumatoid arthritis. Hum 
Gene Ther 2019;30:1008-22.

25. Zheng M, Kuang N, Zeng X, Wang J, Zou Y, Fu Y. Daphnetin induces apoptosis in fibroblast-like synoviocytes from collagen-induced arthritic rats mainly via the mitochondrial pathway. Cytokine 2020;133:155146.

26. Zhao J, Chen B, Peng X, Wang C, Wang K, Han F, et al. Quercetin suppresses migration and invasion by targeting miR-146a/GATA6 axis in fibroblast-like synoviocytes of rheumatoid arthritis. Immunopharmacol Immunotoxicol 2020;42:221-7.

27. Wang Y, Jiao T, Fu W, Zhao S, Yang L, Xu N, et al. miR-410$3 p$ regulates proliferation and apoptosis of fibroblast-like synoviocytes by targeting YY1 in rheumatoid arthritis. Biomed Pharmacother 2019;119:109426.

28. Bao Y, Sun YW, Ji J, Gan L, Zhang CF, Wang CZ, et al. Genkwanin ameliorates adjuvant-induced arthritis in rats through inhibiting JAK/STAT and NF-kappaB signaling pathways. Phytomedicine 2019;63:153036.

29. Myers LK, Rosloniec EF, Cremer MA, Kang AH. Collageninduced arthritis, an animal model of autoimmunity. Life Sci 1997;61:1861-78.

30. Chen Z, Bozec A, Ramming A, Schett G. Anti-inflammatory and immune-regulatory cytokines in rheumatoid arthritis. Nat Rev Rheumatol 2019;15:9-17.

31. Siebert S, Tsoukas A, Robertson J, McInnes I. Cytokines as therapeutic targets in rheumatoid arthritis and other inflammatory diseases. Pharmacol Rev 2015;67:280-309.

32. Saleem A, Saleem M, Akhtar MF, Shahzad M, Jahan S. Polystichum braunii extracts inhibit Complete Freund's adjuvant-induced arthritis via upregulation of I-kappaB, IL-4, and IL-10, downregulation of COX-2, PGE2, IL-1beta, IL-6, NFkappaB, and TNF-alpha, and subsiding oxidative stress. Inflammopharmacology 2020;28:1633-48.

33. Wachsmann D, Sibilia J. Survival in the rheumatoid synovium.
Joint Bone Spine 2011;78:435-7.

34. Kiener HP, Watts GF, Cui Y, Wright J, Thornhill TS, Skold M, et al. Synovial fibroblasts self-direct multicellular lining architecture and synthetic function in three-dimensional organ culture. Arthritis Rheum 2010;62:742-52.

35. Bustamante MF, Garcia-Carbonell R, Whisenant KD, Guma M. Fibroblast-like synoviocyte metabolism in the pathogenesis of rheumatoid arthritis. Arthritis Res Ther 2017;19:110.

36. Du H, Wang Y, Zeng Y, Huang X, Liu D, Ye L, et al. Tanshinone IIA suppresses proliferation and inflammatory cytokine production of synovial fibroblasts from rheumatoid arthritis patients induced by TNF-alpha and attenuates the inflammatory response in AIA mice. Front Pharmacol 2020;11:568.

37. Dong H, Jian P, Yu M, Wang L. Silencing of long noncoding RNA LEF1-AS1 prevents the progression of hepatocellular carcinoma via the crosstalk with microRNA-136-5p/WNK1. J Cell Physiol 2020;235:6548-62.

38. O'Shea JJ, Plenge R. JAK and STAT signaling molecules in immunoregulation and immune-mediated disease. Immunity 2012;36:542-50.

39. Li HW, Zeng HS. Regulation of JAK/STAT signal pathway by miR-21 in the pathogenesis of juvenile idiopathic arthritis. World J Pediatr 2020;16:502-13.

40. Aravilli RK, Vikram SL, Kohila V. Phytochemicals as potential antidotes for targeting NF-kappaB in rheumatoid arthritis. 3 Biotech 2017;7:253.

41. Zhu L, Zhang Z, Xia N, Zhang W, Wei Y, Huang J, et al. Antiarthritic activity of ferulic acid in complete Freund's adjuvant (CFA)-induced arthritis in rats: JAK2 inhibition. Inflammopharmacology 2020;28:463-73.

42. Malemud CJ. The role of the JAK/STAT signal pathway in rheumatoid arthritis. Ther Adv Musculoskelet Dis 2018;10:117-27.

Received for publication: 19 November 2020. Accepted for publication: 12 January 2021.

This work is licensed under a Creative Commons Attribution-NonCommercial 4.0 International License (CC BY-NC 4.0).

(C) Copyright: the Author(s), 2021

Licensee PAGEPress, Italy

European Journal of Histochemistry 2021; 65:3202

doi:10.4081/ejh.2021.3202 